1 Hacettepe Journal of Mathematics and Statistics

$\bigcap$ Volume 47 (6) (2018), 1467-1477

\title{
Abstract Korovkin theory in modular spaces in the sense of power series method
}

\author{
Tuğba Yurdakadim*
}

\begin{abstract}
In this paper, using the power series method we obtain an abstract Korovkin type approximation theorem for a sequence of positive linear operators defined on modular spaces.
\end{abstract}

Keywords: Power series method, modular spaces, abstract Korovkin theory.

Mathematics Subject Classification (2010): Primary 40G10; Secondary 40C15, $41 \mathrm{~A} 36$.

Received : 24.02.2016 Accepted : 06.06.2016 Doi : 10.15672/HJMS.2018.627

\section{Introduction}

The classical Korovkin theorem states the uniform convergence in $C[a, b]$, the space of all continuous real valued functions defined on $[a, b]$ of a sequence of positive linear operators by proving the convergence only on three test functions $\left\{1, x, x^{2}\right\}$. This result lets us to say the convergence with minimum calculations. In fact the key moment of Korovkin's theorem is the proof of Weierstrass's theorem given by Bernstein with the use of Bernstein polynomials [11]. There are also trigonometric versions of this theorem with the test functions $\{1, \cos x, \sin x\}$ and abstract Korovkin type results have also been studied [14, 18]. Later on Korovkin type theorems have been extended in various directions with different aims such as finding other subsets satisfying the same property $\left\{1, x, x^{2}\right\}$, establishing the same results in other function spaces, abstract Banach spaces $[1,2,3]$. Recently some versions of Korovkin type theorems have been proved in modular spaces which include as particular cases $L^{p}$, Orlicz and Musielak-Orlicz spaces [9, 21]. Also more general convergences, for example convergences generated by summability methods, statistical, filter convergence, have also been used for the sequence of operators in order to correct the lack of convergence since it is well known that they provide a nonconvergent sequence to converge $[6,10,12,15,16,17,22,23]$.

In this paper, we give abstract Korovkin theorem in modular spaces in the sense of power series method which includes both Abel and Borel methods. We also give an example which satisfies our theorems.

${ }^{*}$ Department of Mathematics, Hitit University, Çorum, Turkey Email : tugbayurdakadim@hotmail.com 


\section{Notation and definitions}

Let us begin with recalling some basic definitions and notation used in the paper.

Let $\left(p_{j}\right)$ be real sequence with $p_{0}>0$ and $p_{1}, p_{2}, p_{3}, \ldots \geq 0$, and such that the corresponding power series $p(t):=\sum_{j=0}^{\infty} p_{j} t^{j}$ has radius of convergence $R$ with $0<R \leq \infty$. If, for all $t \in(0, R)$,

$$
\lim _{t \rightarrow R^{-}} \frac{1}{p(t)} \sum_{j=0}^{\infty} x_{j} p_{j} t^{j}=L
$$

then we say that $x=\left(x_{j}\right)$ is convergent in the sense of power series method [19, 24]. Note that the power series method is regular if and only if

$$
\lim _{t \rightarrow R^{-}} \frac{p_{j} t^{j}}{p(t)}=0, \text { for each } j \in \mathbb{N}
$$

holds [13]. Throughout the paper we assume that power series method is regular.

Let $G$ be a locally compact Hausdorff topological space, endowed with a uniform structure $\mathcal{U} \subset 2^{G \times G}$ which generates the topology of $G$, see [20]. Let $\mathbb{B}$ be the $\sigma$-algebra of all Borel subsets of $G$, and $\mu: \mathbb{B} \rightarrow \mathbb{R}$ be a positive $\sigma$-finite regular measure. We denote by $L^{0}(G)$ the space of all real-valued $\mu$-measurable functions on $G$ with identification up to sets of measure $\mu$ zero, by $C_{b}(G)$ the space of all continuous real valued and bounded functions on $G$, and by $C_{c}(G)$ the subspace of $C_{b}(G)$ of all functions with compact support on $G$. A functional $\varrho: L^{0}(G) \rightarrow[0, \infty]$ is a modular on $L^{0}(G)$ provided that the following conditions hold:

(i) $\varrho[f]=0$ if and only if $f=0 \mu$-a.e on $G$,

(ii) $\varrho[-f]=\varrho[f]$ for every $f \in L^{0}(G)$,

(iii) $\varrho[\alpha f+\beta g] \leq \varrho[f]+\varrho[g]$ for every $f, g \in L^{0}(G)$ and for any $\alpha, \beta \geq 0$ with $\alpha+\beta=1$.

A modular $\varrho$ is said to be $Q$-quasi convex if there exists a constant $Q \geq 1$ such that the inequality

$$
\varrho[\alpha f+\beta g] \leq Q \alpha \varrho[Q f]+Q \beta \varrho[Q g]
$$

holds for every $f, g \in L^{0}(G), \alpha, \beta \geq 0$ with $\alpha+\beta=1$. In particular if $Q=1$, then $\varrho$ is called convex.

A modular $\varrho$ is said to be $Q$-quasi semiconvex if there exists a constant $Q \geq 1$ such that the inequality

$$
\varrho[a f] \leq Q a \varrho[Q f]
$$

holds for every $f \in L^{0}(G), f \geq 0$ and $a \in(0,1]$. It is clear that every $Q$-quasi convex modular is $Q$-quasi semiconvex. A modular $\varrho$ is said to be monotone if $\varrho[f] \leq \varrho[g]$ for all $f, g \in L^{0}(G)$ with $|f| \leq|g|$.

We now consider some subspaces of $L^{0}(G)$ by means of a modular $\varrho$ as follows

$$
L^{\varrho}(G):=\left\{f \in L^{0}(G): \lim _{\lambda \rightarrow 0^{+}} \varrho[\lambda f]=0\right\}
$$

and

$$
E^{\varrho}(G):=\left\{f \in L^{\varrho}(G): \varrho[\lambda f]<\infty \text { for all } \lambda>0\right\}
$$

are called the modular space generated by $\varrho$ and the space of the finite elements of $L^{\varrho}(G)$, respectively. Observe that if $\varrho$ is $Q$-quasi semiconvex then the space

$$
\left\{f \in L^{0}(G): \varrho[\lambda f]<\infty \text { for some } \lambda>0\right\}
$$

coincides with $L^{\varrho}(G)$. The notions about modulars have been introduced and widely discussed in $[4,5,7,8,9]$. 
Now we define the convergences in the sense of power series method in modular spaces. Let $\left\{f_{j}\right\}$ be a function sequence whose terms belong to $L^{\varrho}(G)$. Then, $\left\{f_{j}\right\}$ is modularly convergent to a function $f \in L^{\varrho}(G)$ in the sense of power series method if and only if

$$
\lim _{t \rightarrow R^{-}} \frac{1}{p(t)} \sum_{j=0}^{\infty} p_{j} t^{j} \varrho\left[\lambda_{0}\left(f_{j}-f\right)\right]=0 \text { for some } \lambda_{0}>0 \text {. }
$$

Also, $\left\{f_{j}\right\}$ is strongly convergent to a function $f \in L^{\varrho}(G)$ in the sense of power series method if and only if

$$
\lim _{t \rightarrow R^{-}} \frac{1}{p(t)} \sum_{j=0}^{\infty} p_{j} t^{j} \varrho\left[\lambda\left(f_{j}-f\right)\right]=0 \text { for every } \lambda>0 .
$$

Recall that $\left\{f_{j}\right\}$ is modularly convergent to a function $f \in L^{\varrho}(G)$ if and only if

$$
\lim _{j \rightarrow \infty} \varrho\left[\lambda_{0}\left(f_{j}-f\right)\right]=0 \text { for some } \lambda_{0}>0,
$$

also $\left\{f_{j}\right\}$ is strongly convergent to a function $f \in L^{\varrho}(G)$ if and only if

$$
\lim _{j \rightarrow \infty} \varrho\left[\lambda\left(f_{j}-f\right)\right]=0 \text { for every } \lambda>0 .
$$

If there exists a constant $M>0$ such that

$$
\varrho[2 u] \leq M \varrho[u]
$$

holds for all $u \geq 0$ then it is said to be that $\varrho$ satisfies the $\Delta_{2}$-condition.

Now we can give the following:

2.1. Theorem. Let $L^{\varrho}(G)$ be a modular space. $\Delta_{2}$-condition is sufficient in order that strong convergence in the sense of power series method and modular convergence in the sense of power series method be equivalent in $L^{\varrho}(G)$.

Proof. Obviously, strong convergence in the sense of power series method of $\left\{f_{j}\right\}$ to $f$ is equivalent to the condition $\lim _{t \rightarrow R^{-}} \frac{1}{p(t)} \sum_{j=0}^{\infty} p_{j} t^{j} \varrho\left[2^{N} \lambda\left(f_{j}-f\right)\right]=0$, for some $\lambda>0$ and all $N=1,2, \ldots$. Let $\left\{f_{j}\right\}$ be modularly convergent to $f$ in the sense of power series method. Then there exists a $\lambda>0$ such that $\lim _{t \rightarrow R^{-}} \frac{1}{p(t)} \sum_{j=0}^{\infty} p_{j} t^{j} \varrho\left[\lambda\left(f_{j}-f\right)\right]=0 . \Delta_{2}$-condition implies by induction that $\varrho\left[2^{N} \lambda\left(f_{j}-f\right)\right] \leq M^{N} \varrho\left[\lambda\left(f_{j}-f\right)\right]$. Therefore we get

$$
\lim _{t \rightarrow R^{-}} \frac{1}{p(t)} \sum_{j=0}^{\infty} p_{j} t^{j} \varrho\left[2^{N} \lambda\left(f_{j}-f\right)\right]=0 .
$$

This completes the proof.

A modular $\varrho$ is said to be finite if $\chi_{A}$, the characteristic function associated with $A$, belongs to $L^{\varrho}(G)$ whenever $A \in \mathbb{B}$ with $\mu(A)<\infty$. A modular $\varrho$ is said to be strongly finite if $\chi_{A}$ belongs to $E^{\varrho}(G)$ for all $A \in \mathbb{B}$ with $\mu(A)<\infty$ and a modular $\varrho$ is said to be absolutely continuous if there is a positive constant $a$ with the property: for all $f \in L^{0}(G)$ with $\varrho[f]<\infty$,

- for each $\varepsilon>0$ there exists a set $A \in \mathbb{B}$ with $\mu(A)<\infty$ and $\varrho\left[a f \chi_{G \backslash A}\right] \leq \varepsilon$,

- for every $\varepsilon>0$ there is a $\delta>0$ with $\varrho\left[a f \chi_{B}\right]$ for every $B \in \mathbb{B}$ with $\mu(B)<\delta$.

Recall that if a modular $\varrho$ is monotone and finite, then we have $C(G) \subset L^{\varrho}(G)$ [7]. In a similar manner, if $\varrho$ is monotone and strongly finite, then $C(G) \subset E^{\varrho}(G)$. 


\section{Modular Korovkin theorem by power series method}

Let $\varrho$ be monotone and finite modular on $L^{0}(G)$. Assume that $D$ is a set satisfying $C_{b}(G) \subset D \subset L^{0}(G)$. Assume further that $T:=\left\{T_{j}\right\}$ is a sequence of positive linear operators from $D$ into $L^{0}(G)$ for which there exists a subset $X_{T} \subset D \bigcap L^{\varrho}(G)$ with $C_{b}(G) \subset X_{T}$ such that the inequality

$$
\limsup _{t \rightarrow R^{-}} \frac{1}{p(t)} \sum_{j=0}^{\infty} p_{j} t^{j} \varrho\left[\lambda\left(T_{j} h\right)\right] \leq P \varrho(\lambda h)
$$

holds for every $h \in X_{T}, \lambda>0$ and for an absolute positive constant $P$. Then it is said that $\varrho$ satisfies the property modified $(\varrho)-(*)$.

Set $e_{0}(t) \equiv 1$ for all $t \in G$, let $e_{i}, i=1,2,3, \ldots, m$, and $a_{i}, i=0,1,2,3, \ldots, m$, be functions in $C_{b}(G)$. Put

$$
P_{s}(t)=\sum_{i=0}^{m} a_{i}(s) e_{i}(t), s, t \in G,
$$

and suppose that $P_{s}(t), s, t \in G$ satisfies the following properties:

- (P1) $P_{s}(s)=0$, for all $s \in G$,

- (P2) for every neighbourhood $U \in \mathcal{U}$ there is a positive real number $\eta$ with $P_{s}(t) \geq \eta$ whenever $s, t \in G,(s, t) \notin U$.

Some examples of $P_{s}$ for which (P1) and (P2) are satisfied have been given in [6].

To give our main theorem, we first give the following result.

3.1. Theorem. Let $\varrho$ be a strongly finite, monotone and $Q$-quasi semiconvex modular. Assume that $e_{i}$ and $a_{i}, i=0,1, \ldots, m$ satisfy properties (P1) and (P2). Let $T_{j}, j \in \mathbb{N}$, be a sequence of positive linear operators satisfying property modified $(\varrho)-(*)$. If

$$
\lim _{t \rightarrow R^{-}} \frac{1}{p(t)} \sum_{j=0}^{\infty} p_{j} t^{j} \varrho\left[\lambda\left(T_{j} e_{i}-e_{i}\right)\right]=0,
$$

for some $\lambda>0$ and $i=0,1, \ldots, m$, then for every $f \in C_{c}(G)$

$$
\lim _{t \rightarrow R^{-}} \frac{1}{p(t)} \sum_{j=0}^{\infty} p_{j} t^{j} \varrho\left[\gamma\left(T_{j} f-f\right)\right]=0,
$$

for some $\gamma>0$. Moreover if

$$
\lim _{t \rightarrow R^{-}} \frac{1}{p(t)} \sum_{j=0}^{\infty} p_{j} t^{j} \varrho\left[\lambda\left(T_{j} e_{i}-e_{i}\right)\right]=0,
$$

for every $\lambda>0$ and $i=0,1, \ldots, m$, then for every $f \in C_{c}(G)$

$$
\lim _{t \rightarrow R^{-}} \frac{1}{p(t)} \sum_{j=0}^{\infty} p_{j} t^{j} \varrho\left[\lambda\left(T_{j} f-f\right)\right]=0
$$

for every $\lambda>0$.

Proof. Let $f \in C_{c}(G)$. Then $f$ is uniformly continuous and bounded on $G$ since $G$ is endowed with $\mathcal{U}$ uniformity. Let $\varepsilon>0$. Without losing anything from generality we can choose $0<\varepsilon \leq 1$. From the uniform continuity of $f$, there exists a set $U \in \mathcal{U}$ such that

$$
|f(s)-f(t)| \leq \varepsilon, \quad s, t \in G, \quad(s, t) \in U .
$$


For every $s, t \in G$ let $P_{s}(t)$ be as in (3.2) and let $\eta>0$ satisfy condition (P2). If $M=\sup _{t \in G}|f(t)|$, for $s, t \in G,(s, t) \notin U$ we have

$$
|f(s)-f(t)| \leq 2 M \leq \frac{2 M}{\eta} P_{s}(t) .
$$

For every $s, t \in G$, we obtain

$$
|f(s)-f(t)| \leq 2 M \leq \varepsilon+\frac{2 M}{\eta} P_{s}(t) .
$$

Therefore for every $s, t \in G$, we get

$$
-\varepsilon-\frac{2 M}{\eta} P_{s}(t) \leq f(s)-f(t) \leq \varepsilon+\frac{2 M}{\eta} P_{s}(t) .
$$

Since $T_{j}$ is a linear positive operator, using (3.3) for each $j \in \mathbb{N}$ and every $s \in G$ we have

$$
\begin{aligned}
-\varepsilon\left(T_{j} e_{0}\right)(s)-\frac{2 M}{\eta}\left(T_{j} P_{s}\right)(s) & \leq f(s)\left(T_{j} e_{0}\right)(s)-\left(T_{j} f\right)(s) \\
& \leq \varepsilon\left(T_{j} e_{0}\right)(s)+\frac{2 M}{\eta}\left(T_{j} P_{s}\right)(s)
\end{aligned}
$$

and hence

$$
\begin{aligned}
\left|\left(T_{j} f\right)(s)-f(s)\right| & \leq\left|\left(T_{j} f\right)(s)-f(s)\left(T_{j} e_{0}\right)(s)\right|+\left|f(s)\left(T_{j} e_{0}\right)(s)-f(s)\right| \\
& \leq \varepsilon\left(T_{j} e_{0}\right)(s)+\frac{2 M}{\eta}\left(T_{j} P_{s}\right)(s)+M\left|\left(T_{j} e_{0}\right)(s)-e_{0}(s)\right| .
\end{aligned}
$$

Let $\gamma>0$. By applying the modular $\varrho$, from the above inequality, we have

$$
\begin{aligned}
\varrho\left[\gamma\left(T_{j} f-f\right)\right] & \leq \varrho\left[3 \gamma \varepsilon\left(T_{j} e_{0}\right)\right]+\varrho\left[3 \gamma M\left(T_{j} e_{0}-e_{0}\right)\right]+\varrho\left[6 \gamma \frac{M}{\eta}\left(T_{j} P_{(.)}\right)(.)\right] \\
& =J_{1}+J_{2}+J_{3}, \text { for each } j \in \mathbb{N} .
\end{aligned}
$$

So to prove the theorem it is sufficient to show that there exists a positive real number $\gamma$ such that $\lim _{t \rightarrow R^{-}} \frac{1}{p(t)} \sum_{j=0}^{\infty} p_{j} t^{j} \varrho\left[\gamma\left(T_{j} f-f\right)\right]=0$. From hypothesis there exists a $\lambda>0$ such that for each $i=0,1, \ldots, m$

$$
\lim _{t \rightarrow R^{-}} \frac{1}{p(t)} \sum_{j=0}^{\infty} p_{j} t^{j} \varrho\left[\lambda\left(T_{j} e_{i}-e_{i}\right)\right]=0 .
$$

For each $i=0,1, \ldots, m$ and $s \in G$, choose $N>0$ and $\gamma>0$ such that $\left|a_{i}(s)\right| \leq N$ and $\max \left\{3 \gamma M, 6 \gamma \frac{M}{\eta}(m+1) N\right\} \leq \lambda$. By considering the property (P1), for each $j \in \mathbb{N}$ we get

$$
\begin{aligned}
J_{3}=\varrho\left[6 \gamma \frac{M}{\eta}\left(T_{j} P_{(.)}\right)(.)\right] & =\varrho\left[6 \gamma \frac{M}{\eta}\left(T_{j} P_{(.)}\right)(.)-P_{(.)}(.)\right] \\
& \leq \sum_{i=0}^{m} \varrho\left[6 \gamma \frac{M}{\eta}(m+1) N\left(T_{j} e_{i}-e_{i}\right)\right] \\
& \leq \sum_{i=0}^{m} \varrho\left[\lambda\left(T_{j} e_{i}-e_{i}\right)\right] .
\end{aligned}
$$

Hence we obtain

$$
\lim _{t \rightarrow R^{-}} \frac{1}{p(t)} \sum_{j=0}^{\infty} p_{j} t^{j} J_{3}=0 .
$$


Moreover from choosing $\lambda$ and $\gamma$, it is clear that $\lim _{t \rightarrow R^{-}} \frac{1}{p(t)} \sum_{j=0}^{\infty} p_{j} t^{j} J_{2}=0$. Since $\varrho$ is $Q$-quasi semiconvex and $0<\varepsilon \leq 1$, we have

$$
\varrho\left[3 \gamma \varepsilon e_{0}\right] \leq Q \varepsilon \varrho\left[3 \gamma Q e_{0}\right] .
$$

If the property modified $(\varrho)-(*)$ is considered in $(3.5),(3.7)$, we get

$$
\begin{aligned}
0 \leq \limsup _{t \rightarrow R^{-}} \frac{1}{p(t)} \sum_{j=0}^{\infty} p_{j} t^{j} \varrho\left[\gamma\left(T_{j} f-f\right)\right] & \leq \limsup _{t \rightarrow R^{-}} \frac{1}{p(t)} \sum_{j=0}^{\infty} p_{j} t^{j} \varrho\left[3 \gamma \varepsilon\left(T_{j} e_{0}\right)\right] \\
& \leq P \varrho\left[3 \gamma \varepsilon e_{0}\right] \leq P Q \varepsilon \varrho\left[3 \gamma Q e_{0}\right]
\end{aligned}
$$

by applying the limit superior. Since $\varepsilon$ is arbitrary positive real number and $\varrho$ is strongly finite from (3.8) we have

$$
\limsup _{t \rightarrow R^{-}} \frac{1}{p(t)} \sum_{j=0}^{\infty} p_{j} t^{j} \varrho\left[\gamma\left(T_{j} f-f\right)\right]=0,
$$

and hence $\left\{T_{j} f\right\}$ is modularly convergent to $f$ in the sense of power series method in $L^{\varrho}(G)$ by using the properties of limit in the sense of power series method and limit superior. The second part can be proved similarly to the first one.

Now we can give our main theorem.

3.2. Theorem. Let $\varrho$ be a strongly finite, monotone, absolutely continuous and Q-quasi semiconvex modular on $L^{0}(G)$. Let $T_{j}, j \in \mathbb{N}$, be a sequence of positive linear operators satisfying property modified $(\varrho)-(*)$. If

$$
\lim _{t \rightarrow R^{-}} \frac{1}{p(t)} \sum_{j=0}^{\infty} p_{j} t^{j} \varrho\left[\lambda\left(T_{j} e_{i}-e_{i}\right)\right]=0
$$

for every $\lambda>0$ and $i=0,1, \ldots, m$, then for every $f \in L^{\varrho}(G) \cap D$ with $f-C_{b}(G) \subset X_{T}$,

$$
\lim _{t \rightarrow R^{-}} \frac{1}{p(t)} \sum_{j=0}^{\infty} p_{j} t^{j} \varrho\left[\gamma\left(T_{j} f-f\right)\right]=0
$$

for some $\gamma>0$ where $X_{T}$ and $D$ are as before.

Proof. Let $f \in L^{\varrho}(G) \cap D$ such that $f-C_{b}(G) \subset X_{T}$. From Proposition 3.2 of [6] there exist a $\lambda>0$ and a sequence $\left\{f_{m}\right\}$ in $C_{c}(G)$ such that $\varrho[3 \lambda f]<\infty$ and $\lim _{m} \varrho\left[3 \lambda\left(f_{m}-f\right)\right]=$ 0 . Take arbitrary fixed $\varepsilon>0$ and choose a positive integer $\bar{m}$ such that

$$
\varrho\left[3 \lambda\left(f_{\bar{m}}-f\right)\right] \leq \varepsilon
$$

For each $j \in \mathbb{N}$, we have

$$
\varrho\left[\lambda\left(T_{j} f-f\right)\right] \leq \varrho\left[3 \lambda\left(T_{j} f-T_{j} f_{\bar{m}}\right)\right]+\varrho\left[3 \lambda\left(T_{j} f_{\bar{m}}-T_{j} f_{\bar{m}}\right)\right]+\varrho\left[3 \lambda\left(f_{\bar{m}}-f\right)\right] .
$$

Using the similar technique in the previous theorem, we obtain

$$
0=\lim _{t \rightarrow R^{-}} \frac{1}{p(t)} \sum_{j=0}^{\infty} p_{j} t^{j} \varrho\left[3 \lambda\left(T_{j} f_{\bar{m}}-f_{\bar{m}}\right)\right]=\limsup _{t \rightarrow R^{-}} \frac{1}{p(t)} \sum_{j=0}^{\infty} p_{j} t^{j} \varrho\left[3 \lambda\left(T_{j} f_{\bar{m}}-f_{\bar{m}}\right)\right] .
$$

From the property modified $(\varrho)-(*)$, there exists an $P>0$ such that

$$
\lim _{t \rightarrow R^{-}} \frac{1}{p(t)} \sum_{j=0}^{\infty} p_{j} t^{j} \varrho\left[3 \lambda\left(T_{j} f-T_{j} f_{\bar{m}}\right)\right] \leq P \varrho\left[3 \lambda\left(f-f_{\bar{m}}\right)\right] \leq P \varepsilon
$$


From (3.9)-(3.11) and subadditivity of the operator lim sup we have

$$
0 \leq \limsup _{t \rightarrow R^{-}} \frac{1}{p(t)} \sum_{j=0}^{\infty} p_{j} t^{j} \varrho\left[\lambda\left(T_{j} f-f\right)\right] \leq \varepsilon(P+1) .
$$

From (3.12) and arbitrariness of $\varepsilon$ we get

$$
\limsup _{t \rightarrow R^{-}} \frac{1}{p(t)} \sum_{j=0}^{\infty} p_{j} t^{j} \varrho\left[\lambda\left(T_{j} f-f\right)\right]=0 .
$$

Thus one can get

$$
\lim _{t \rightarrow R^{-}} \frac{1}{p(t)} \sum_{j=0}^{\infty} p_{j} t^{j} \varrho\left[\lambda\left(T_{j} f-f\right)\right]=0
$$

and this completes the proof.

\section{Concluding remarks}

Take $G=[0,1]$ and let $\varphi:[0, \infty) \rightarrow[0, \infty)$ be a continuous function for which the following conditions hold:

- $\varphi$ is convex,

- $\varphi(0)=0, \varphi(u)>0$ for $u>0$ and $\lim _{u \rightarrow+\infty} \varphi(u)=\infty$.

Here, consider the functional $\varrho^{\varphi}$ on $L^{0}(G)$ defined by

$$
\varrho^{\varphi}[f]:=\int_{0}^{1} \varphi(|f(x)|) d x, \text { for } f \in L^{0}(G) .
$$

In this case, $\varrho^{\varphi}$ is a convex modular on $L^{0}(G)$ (see [7]). Consider the Orlicz space generated by $\varphi$ as follows

$$
L_{\varphi}^{\varrho}(G):=\left\{f \in L^{0}(G): \varrho^{\varphi}[\lambda f]<\infty \text { for some } \lambda>0\right\} .
$$

Then, consider the following classical Bernstein-Kantorovich operator $\mathbb{U}:=\left\{U_{j}\right\}$ on the space $L_{\varphi}^{\varrho}(G)$ (see [7]) which is defined by

$$
U_{j}(f ; x):=\sum_{k=0}^{j}\left(\begin{array}{l}
j \\
k
\end{array}\right) x^{k}(1-x)^{j-k}(j+1) \int_{\frac{k}{j+1}}^{\frac{k+1}{j+1}} f(t) d t ; x \in G .
$$

Observe that the operators $U_{j}$ map the Orlicz space $L_{\varphi}^{\varrho}(G)$ into itself. Moreover, it is also known that the property $\limsup _{j \rightarrow \infty} \varrho\left[\lambda\left(U_{j} h\right)\right] \leq M \varrho[\lambda h]$ is satisfied with the choice of $X_{U}:=L_{\varphi}^{\varrho}(G)$ and for every function $f \in L_{\varphi}^{\varrho}(G)$ such that $f-g \in X_{U}$ for every $g \in C_{b}(G),\left\{U_{j} f\right\}$ is modularly convergent to $f$. Using the operators $\left\{U_{j} f\right\}$ define the sequence of positive linear operators $V:=V_{j}$ on $L_{\varphi}^{\varrho}(G)$ as follows:

$$
V_{j}(f ; x)=\left(1+s_{j}\right) U_{j}(f ; x), \text { for } f \in L_{\varphi}^{\varrho}(G), x \in[0,1] \text { and } j \in \mathbb{N},
$$

where $s_{j}=1, j$ is square and 0 otherwise. Also let $R=1, p(t)=\frac{1}{1-t}$ and for $j \in \mathbb{N}^{0}$, $p_{j}=1$. In this case the power series method coincides with Abel method. Note that 
$\left\{s_{j}\right\}$ is convergent to 0 in the sense of power series method. By Lemma 5.1 of [7], for every $h \in X_{V}:=L_{\varphi}^{\varrho}(G)$, all $\lambda>0$ and for an absolute positive constant $P$, we get

$$
\begin{aligned}
\varrho^{\varphi}\left[\lambda V_{j} h\right]=\varrho^{\varphi}\left[\lambda\left(1+s_{j}\right) U_{j} h\right] & \leq \varrho^{\varphi}\left[2 \lambda U_{j} h\right]+\varrho^{\varphi}\left[2 \lambda s_{j} U_{j} h\right] \\
& =\varrho^{\varphi}\left[2 \lambda U_{j} h\right]+s_{j} \varrho^{\varphi}\left[2 \lambda U_{j} h\right] \\
& =\left(1+s_{j}\right) \varrho^{\varphi}\left[2 \lambda U_{j} h\right] \leq\left(1+s_{j}\right) P \varrho^{\varphi}[2 \lambda h] .
\end{aligned}
$$

Then, we get

$$
\limsup _{t \rightarrow R^{-}} \frac{1}{p(t)} \sum_{j=0}^{\infty} p_{j} t^{j} \varrho^{\varphi}\left[\lambda V_{j} h\right] \leq P \varrho^{\varphi}[2 \lambda h] .
$$

Now, we show that conditions in the Theorem 3.2 holds. First note that

$$
\begin{aligned}
& V_{j}\left(e_{0} ; x\right)=1+s_{j} \\
& V_{j}\left(e_{1} ; x\right)=\left(1+s_{j}\right)\left\{\frac{j x}{j+1}+\frac{1}{2(j+1)}\right\} \\
& V_{j}\left(e_{2} ; x\right)=\left(1+s_{j}\right)\left\{\frac{j(j-1) x^{2}}{(j+1)^{2}}+\frac{2 j x}{(j+1)^{2}}+\frac{1}{3(j+1)^{2}}\right\}
\end{aligned}
$$

where $e_{i}(t)=t^{i}$. So for any $\lambda>0$, we can see, that

$$
\lambda\left|V_{j}\left(e_{0} ; x\right)-e_{0}(x)\right|=\lambda\left|1+s_{j}-1\right|=\lambda s_{j},
$$

which implies

$$
\varrho^{\varphi}\left[\lambda\left(V_{j}\left(e_{0}\right)-e_{0}\right)\right]=\varrho^{\varphi}\left[\lambda s_{j}\right]=\int_{0}^{1} \varphi\left(\lambda s_{j}\right) d x=\varphi\left(\lambda s_{j}\right)=s_{j} \varphi(\lambda)
$$

because of the definition of $\left\{s_{j}\right\}$. Since $\left\{s_{j}\right\}$ is convergent to 0 in the sense of power series method

$$
\lim _{t \rightarrow R^{-}} \frac{1}{p(t)} \sum_{j=0}^{\infty} p_{j} t^{j} \varrho^{\varphi}\left[\lambda V_{j}\left(e_{0}\right)-e_{0}\right]=\lim _{t \rightarrow R^{-}} \frac{1}{p(t)} \sum_{j=0}^{\infty} p_{j} t^{j} s_{j} \varphi(\lambda)=0,
$$

for every $\lambda>0$. Also

$$
\begin{aligned}
\lambda\left|V_{j}\left(e_{1} ; x\right)-e_{1}(x)\right| & =\lambda\left|x\left(\frac{j}{j+1}+\frac{j s_{j}}{j+1}-1\right)+\frac{1}{2(j+1)}+\frac{s_{j}}{2(j+1)}\right| \\
& \leq \lambda\left\{\frac{3}{2(j+1)}+s_{j}\left(\frac{2 j+1}{2(j+1)}\right)\right\}
\end{aligned}
$$

we may write that

$$
\begin{aligned}
\varrho^{\varphi}\left[\lambda\left(V_{j}\left(e_{1}\right)-e_{1}\right)\right] & \leq \varrho^{\varphi}\left[\lambda\left\{s_{j}\left(\frac{2 j+1}{2(j+1)}\right)+\frac{3}{2(j+1)}\right\}\right] \\
& \leq s_{j} \varrho^{\varphi}\left[\lambda\left(\frac{2 j+1}{j+1}\right)\right]+\varrho^{\varphi}\left[\frac{3 \lambda}{j+1}\right]
\end{aligned}
$$

by the definitions of $\left\{s_{j}\right\}$ and $\varrho^{\varphi}$. Since $\left\{\frac{2 j+1}{j+1}\right\}$ is convergent, there exists a constant $M>0$ such that $\left\{\frac{2 j+1}{j+1} \leq M\right\}$, for every $j \in \mathbb{N}$. Then using the monotonicity of $\varrho^{\varphi}$, we have

$$
\varrho^{\varphi}\left[\lambda \frac{2 j+1}{j+1}\right] \leq \varrho^{\varphi}[\lambda M]
$$


for any $\lambda>0$, which implies

$$
\varrho^{\varphi}\left[\lambda\left(V_{j}\left(e_{1}\right)-e_{1}\right)\right] \leq s_{j} \varrho^{\varphi}[\lambda M]+\varrho^{\varphi}\left[\frac{3 \lambda}{j+1}\right]=s_{j} \varphi(\lambda M)+\varphi\left(\frac{3 \lambda}{j+1}\right) .
$$

Since $\varphi$ is continuous, we have $\lim _{j} \varphi\left(\frac{3 \lambda}{j+1}\right)=\varphi\left(\lim _{j} \frac{3 \lambda}{j+1}\right)=\varphi(0)=0$. So we get $\varphi\left(\frac{3 \lambda}{j+1}\right)$ is convergent to 0 in the sense of power series method. Using this and by the definition of $\left\{s_{j}\right\}$, we obtain

$$
\begin{aligned}
\lim _{t \rightarrow R^{-}} \frac{1}{p(t)} \sum_{j=0}^{\infty} p_{j} t^{j} \varrho^{\varphi}\left[\lambda V_{j}\left(e_{1}\right)-e_{1}\right] & \leq \lim _{t \rightarrow R^{-}} \frac{1}{p(t)} \sum_{j=0}^{\infty} p_{j} t^{j}\left[s_{j} \varphi(\lambda M)+\varphi\left(\frac{3 \lambda}{j+1}\right)\right] \\
& =\varphi(\lambda M) \lim _{t \rightarrow R^{-}} \frac{1}{p(t)} \sum_{j=0}^{\infty} p_{j} t^{j} s_{j} \\
& +\lim _{t \rightarrow R^{-}} \frac{1}{p(t)} \sum_{j=0}^{\infty} p_{j} t^{j} \varphi\left(\frac{3 \lambda}{j+1}\right) \\
& =0
\end{aligned}
$$

Finally, since

$$
\begin{aligned}
& \lambda\left|V_{j}\left(e_{2} ; x\right)-e_{2}(x)\right| \\
& =\lambda \mid x^{2} \frac{j(j-1)}{(j+1)^{2}}+\frac{2 j x}{(j+1)^{2}}+\frac{1}{3(j+1)^{2}}+s_{j} \frac{j(j-1) x^{2}}{(j+1)^{2}}+s_{j} \frac{2 j x}{(j+1)^{2}} \\
& +s_{j} \frac{1}{3(j+1)^{2}}-x^{2} \mid \\
& \leq \lambda\left\{\frac{15 j+4}{3(j+1)^{2}}+s_{j}\left(\frac{3 j^{2}+3 j+1}{3(j+1)^{2}}\right)\right\} .
\end{aligned}
$$

Since $\left\{\frac{3 j^{2}+3 j+1}{3(j+1)^{2}}\right\}$ is convergent, there exists a constant $K>0$ such that $\left|\frac{3 j^{2}+3 j+1}{3(j+1)^{2}}\right| \leq K$, for every $j \in \mathbb{N}$. Then using the monotonicity of $\varrho^{\varphi}$ and the definition of $\left\{s_{j}\right\}$, we have

$$
\begin{aligned}
\varrho^{\varphi}\left[\lambda\left(V_{j}\left(e_{2}\right)-e_{2}\right)\right] & \leq \varrho^{\varphi}\left[2 \lambda\left(\frac{15 j+4}{3(j+1)^{2}}\right)\right]+\varrho^{\varphi}\left[2 \lambda s_{j}\left(\frac{3 j^{2}+3 j+1}{3(j+1)^{2}}\right)\right] \\
& \leq \varrho^{\varphi}\left[\lambda\left(\frac{30 j+8}{3(j+1)^{2}}\right)\right]+\varrho^{\varphi}\left[2 \lambda s_{j} K\right]
\end{aligned}
$$

which yields

$$
\varrho^{\varphi}\left[\lambda\left(V_{j}\left(e_{2}\right)-e_{2}\right)\right] \leq \varphi\left[\lambda\left(\frac{30 j+8}{3(j+1)^{2}}\right)\right]+s_{j} \varphi(2 \lambda K) .
$$

Since $\varphi$ is continuous, we have $\lim _{j} \varphi\left(\lambda \frac{30 j+8}{3(j+1)^{2}}\right)=\varphi\left(\lambda \lim _{j} \frac{30 j+8}{3(j+1)^{2}}\right)=\varphi(0)=0$. So we get $\varphi\left(\lambda \frac{30 j+8}{3(j+1)^{2}}\right)$ is convergent to 0 in the sense of power series method. Using this and by the definition of $\left\{s_{j}\right\}$, we obtain

$$
\lim _{t \rightarrow R^{-}} \frac{1}{p(t)} \sum_{j=0}^{\infty} p_{j} t^{j} \varrho^{\varphi}\left[\lambda V_{j}\left(e_{2}\right)-e_{2}\right]=0, \text { for every } \lambda>0 .
$$

So we can say that our sequence $V:=\left\{V_{j}\right\}$ satisfies all assumptions of Theorem 3.2. Therefore we conclude that

$$
\lim _{t \rightarrow R^{-}} \frac{1}{p(t)} \sum_{j=0}^{\infty} p_{j} t^{j} \varrho^{\varphi}\left[\lambda_{0} V_{j}(f)-f\right]=0, \text { for some } \lambda_{0}>0
$$


holds for every $f \in L_{\varphi}^{\varrho}(G)$ such that $f-g \in X_{V}$ for every $g \in C_{b}(G)$. However since $\left\{s_{j}\right\}$ is not convergent to zero, it is clear that $\left\{V_{j}(f)\right\}$ is not modularly convergent to $f$.

Note that

- in the case of $R=1, p(t)=\frac{1}{1-t}$ and for $j \in \mathbb{N}^{0}, p_{j}=1$ the power series method coincides with Abel method which is a sequence-to-function transformation,

- in the case of $R=\infty, p(t)=e^{t}$ and for $j \in \mathbb{N}^{0}, p_{j}=\frac{1}{j !}$ the power series method coincides with Borel method.

We can therefore give all of the theorems of this paper for Abel and Borel convergences.

\section{References}

[1] Altomare, F., Korovkin-type theorems and approximation by positive linear operators, Surveys in Approximation Theory 5.13, 2010.

[2] Altomare, F. and Diomede, S., Contractive Korovkin subsets in weighted spaces of continuous functions, Rend. Circ. Mat. Palermo 50, 547-568, 2001.

[3] Atlihan, Ö. G. and Taş, E., An abstract version of the Korovkin theorem via A-summation process, Acta. Math. Hungar. 145, 360-368, 2015.

[4] Bardaro, C. Boccuto, A. Dimitriou, X. and Mantellini, I., A Korovkin theorem in multivariate modular function spaces, J. Func. Spaces Appl., 7, 105-120, 2009.

[5] Bardaro, C. Boccuto, A. Dimitriou, X. and Mantellini, I., Modular filter convergence theorems for abstract sampling-type operators, Appl. Anal. 92, 2404-2423, 2013.

[6] Bardaro, C. Boccuto, A. Dimitriou, X. and Mantellini, I., Abstract Korovkin-type theorems in modular spaces and applications, Cent. Eur. J. Math., 11, 1774-1784, 2013.

[7] Bardaro, C. and Mantellini, I., Korovkin's theorem in modular spaces, Comment. Math. 47, 239-253, 2007.

[8] Bardaro, C. and Mantellini, I., Multivariate moment type operators: approximation properties in Orlicz spaces, J. Math. Ineq. 2, 247-259, 2008.

[9] Bardaro, C. Musielak, J. and Vinti, G., Nonlinear Integral Operators and Applications, De Gruyter Ser. Nonlinear Anal. Appl. 9, Walter de Gruyter, Berlin, 2003.

[10] Belen, C. and Yildirim, M., Statistical approximation in multivariate modular function spaces, Comment. Math. 51, 39-53, 2011.

[11] Bernstein, F., Über eine Anwendung der Mengenlehre auf ein der Theorie der säkularen Störungen herrührendes Problem, Math. Ann., 71, 417-439, 1912.

[12] Boccuto, A. and Dimitriou, X., Modular filter convergence theorems for Urysohn integral operators and applications, Acta Math. Sinica, 29, 1055-1066, 2013.

[13] Boos, J., Classical and Modern Methods in Summability, Oxford University Press, 2000.

[14] Butzer, P. L. and Berens, H., Semi-groups of operators and approximation, Die Grundlehren der Mathematischen Wissenschaften, 145, Springer, New York, 1967.

[15] Gadjiev, A. D. and Orhan, C., Some approximation theorems via statistical convergence, Rocky Mountain J. Math. 32, 129-138, 2002.

[16] Karakus, S. and Demirci, K., Matrix summability and Korovkin type approximation theorem on modular spaces, Acta Math. Univ. Commenianae, 2, 281-292, 2010.

[17] Karakus, S. Demirci, K. Duman, O., Statistical approximation by positive linear operators on modular spaces, Positivity, 14, 321-334, 2010.

[18] Korovkin, P. P., Linear Operators and Approximation Theory, Hindustan Publ. Co., Delhi, 1960.

[19] Kratz, W. and Stadtmüller, U., Tauberian theorems for $J_{p}$-summability, J. Math. Anal. Appl. 139, 362-371, 1989.

[20] Kuratowski, K., Topology I-II, Academic Press/PWN, New York-London/Warsaw, 19661968.

[21] Musielak, J., Orlicz Spaces and Modular Spaces, Lecture Notes in Math., 1034, Springer, Berlin, 1983.

[22] Musielak, J. and Orlicz, W., On modular spaces, Studia Math. 18, 49-65, 1959. 
[23] Özgüç, I. and Taş, E., A Korovkin-type approximation theorem and power series method, Results. Math. 69, 497-504, 2016.

[24] Stadtmüller, U. and Tali, A., On certain families of generalized Nörlund methods and power series methods, J. Math. Anal. Appl. 238, 44-66, 1999. 